\title{
Análisis del bienestar psicológico en grupos en riesgo de exclusión social
}

\author{
Andrés Escarbajal-Frutos ${ }^{1 *}$, Tomás Izquierdo-Rus ${ }^{2}$ y Olivia López-Martínez ${ }^{3}$ \\ ${ }^{1}$ Departamento de Didáctica y Organización Escolar. Universidad de Murcia. \\ ${ }^{2}$ Departamento de Métodos de Investigación y Diagnóstico en Educación. Universidad de Murcia. \\ ${ }^{3}$ Departamento de Psicología Evolutiva y de la Educación. Universidad de Murcia.
}

\begin{abstract}
Resumen: En el presente artículo analizamos el bienestar psicológico, como un estado integral de salud psicológico, social y laboral, en los grupos con mayor riesgo de exclusión social, entendida ésta como la falta de participación social, económica, política y cultural. Para ello utilizamos una metodología descriptiva de corte transversal. Destacamos que, de un total de 157 participantes, la mayor parte de ellos presentaron un nivel medio de satisfacción en sus vidas; evidenciaron diferencias estadísticamente no significativas en función del sexo; y al comparar los grupos de edad, resultó que no encontramos diferencias significativas en bienestar psicológico en función del grupo de pertenencia. Con esta investigación pretendemos mejorar sus condiciones socioeconómicas, psicológicas y personales, así como proponer alternativas de actuación para construir una sociedad más equitativa e inclusiva.
\end{abstract}

Palabras clave: Exclusión; bienestar psicológico; salud; inclusión.

\section{Introducción}

Al menos las dos últimas décadas han estado presididas por la economía como principal dimensión de la realidad y como paradigma ideológico imperante. Hoy ese modelo está fuertemente cuestionado por la regresión social que ha conllevado el aumento de las desigualdades, el incremento de los índices de marginación, etc., por lo que se ha pensado en situar a los programas psicoeducativos en la vanguardia de los nuevos modelos sociales buscando el bienestar de todos, pero especialmente de los grupos en riesgo de exclusión social (Hessel, 2011).

Desde la psicología (Seligman y Csikszentmihalyi, 2000) se ha comprobado que el estudio de los rasgos positivos, las fortalezas del carácter, las emociones positivas, las experiencias vividas, la familia (Kennedy y Kramer, 2008; Kevin, 2009), la escuela, el contexto y las relaciones positivas, etc., (Peterson, 2006) producen efectos y cambios en el bienestar de los individuos y en sus procesos de inclusión social. No puede existir el bienestar individual sin el bienestar social (Keyes y López, 2002), pues el estado de felicidad plena no solo se consigue con el estado de satisfacción de una persona consigo misma, sino con todo lo que le rodea (Hervás, 2009); por ello presentamos este estudio donde se analiza el bienestar psicológico de los grupos en riesgo de exclusión.

La globalización económica ha creado en las ciudades grandes bolsas de desempleo y marginación, empleo precario, subempleo y pluriempleo..., condiciones que influyen negativamente en el equilibrio psicosocial de los grupos y personas en riesgo de exclusión social (Portera, 2006), pero también en grupos que 'se sentían' alejados de esas situacio-

* Dirección para correspondencia [Correspondence address]: Andrés Escarbajal Frutos. Departamento de Didáctica y Organización Escolar. Universidad de Murcia, Campus Universitario de Espinardo s/n. 30100 Murcia (España). E-mail: andreses@um.es

\begin{abstract}
Title: Analysis of psychological well-being in groups at risk of social exclusion.

Abstract: In this work analyze the psychological well-being as an integral state of health psychological, social and labor, in the groups with risk of social exclusion, understood as the need social, economic, political and cultural participation. In this study use a transversal descriptive methodology. It emphasizes that from a total of 157 participants, most of them presented a medium level of satisfaction in their lives; be found differences statistically not significant based on sex; and to compare the age groups, we found no significant differences in psychological well-being depending on the membership group. This research aims to improve their socioeconomic, psychological and personal conditions, as well as to propose alternatives for action to make a more equitable and inclusive society. Key words: Exclusion; psychology well being; health; inclusion.
\end{abstract}

nes, más concretamente en los grupos de jóvenes, en los mayores de 40 años y en las mujeres. Esos grupos perciben que, cada vez más, se ven limitadas sus condiciones para la convivencia normalizada, el trabajo, las relaciones sociales, la vivienda e incluso, en algunos casos, la manutención. En ocasiones, la carencia de participación, de funciones y responsabilidades asociadas con un trabajo puede ser un indicador de exclusión laboral entre tus compañeros y afecta al bienestar psicológico del individuo (Dolan, García y Díez (2005).

Los excluidos tradicionales eran personas o grupos que habían estado siempre en los márgenes de la sociedad, sin entrar en los circuitos del empleo y la sociabilidad, cuya situación se reproducía de generación en generación y que normalmente eran llamados pobres. Pero actualmente hay 'nuevos pobres', titulares de una pobreza que surge como consecuencia de una degradación con respecto a situaciones anteriores de esas misma personas. $\mathrm{Y}$ también hay personas que, no siendo pobres ni excluidos, son vulnerables por la precariedad de su situación social. De ahí que Castel (2004) distinga entre una zona de integración en la que viven personas con trabajo y sociabilidad estables y con expectativas de continuar en esa situación; una zona de vulnerabilidad en la que se encuentran las personas que viven constantemente (o al menos gran parte de su trayectoria vital) en situaciones de inestabilidad; y finalmente una tercera zona, la de la exclusión propiamente dicha, en la que pueden caer tanto los que parecían integrados como los vulnerables, y que significa estar alejados de la sociabilidad, del trabajo e incluso en muchos casos de la condición de ciudadanía.

Necesitamos, por tanto, una nueva generación de derechos sociales que atiendan al principio de dignidad de todo ser humano. Derechos que tengan en cuenta las desigualdades reales y el acceso a las necesidades básicas, aspectos que inciden de manera muy directa a la hora de desarrollar una vida digna (Herrera, 2005). Tener en cuenta estos postulados 
nos llevaría a la creación de una sociedad inclusiva "que busca la equidad y la lucha por combatir la desigualdad, la injusticia y toda forma de discriminación que suponga una agresión a los derechos humanos" (De Haro, 2009, p. 220).

A tenor de las consideraciones anteriores, en el presente artículo damos cuenta de un estudio que analiza el bienestar psicosocial de los grupos en riesgo de exclusión, más concretamente jóvenes, mayores de 40 años y mujeres, para mejorar sus condiciones socioeconómicas y psicopersonales y proponer, en su caso, alternativas de actuación para construir una sociedad más equitativa e inclusiva. En ese estudio participaron 157 personas de Andalucía y utilizamos una metodología descriptiva de corte transversal, como veremos más adelante.

\section{Vulnerabilidad y exclusión social}

Bajo el rótulo de la exclusión social se cataloga a una serie heterogénea de personas y grupos marginados que pone en cuestión el tan cacareado progreso de la sociedad. Por exclusión social entendemos la falta de participación social, económica, política y cultural; como por ejemplo, dificultades de acceso laboral, dificultad para adquirir una vivienda digna, no recibir una educación de calidad, estar excluido de recibir una atención sanitaria primaria, etc., cuestiones tan relevantes y actuales que la Unión Europea proclamó el 2010 como el año europeo de la lucha contra la pobreza y la exclusión social. En nuestro estudio, y dado que la exclusión se da un proceso de privaciones de variables económicas, sociales, laborales, culturales, educativas y de género, consideramos necesario el análisis de los mecanismos que conducen a ella desde la perspectiva de las personas pertenecientes a grupos en situación de vulnerabilidad (Barton, 2008).

De todas formas, somos conscientes de que el concepto de vulnerabilidad social es confundido y mal utilizado como sinónimo de los conceptos de pobreza y exclusión; y no son lo mismo. La pobreza expresa una condición de necesidad resultante de insuficientes ingresos, mientras que la vulnerabilidad tiene que ver con la indefensión, la inseguridad y la exposición a riesgos provocados por diversos acontecimientos y circunstancias, al tiempo que incorpora a los análisis los recursos de cada grupo para afrontar esos riesgos. La inseguridad e indefensión que caracteriza a la vulnerabilidad no es atribuible en exclusividad a la insuficiencia de ingresos, más propia de la pobreza. Como escribe, Pizarro (2001, p. 8) "existen otras dimensiones de la vida social, y no sólo el trabajo, en las que el riesgo y la inseguridad son dominantes". Explica este autor que, cuando hablamos de pobreza, estamos calificando con ciertos atributos a un colectivo o grupo social de forma descriptiva sin penetrar en los procesos que causan tales situaciones; sin embargo, cuando hablamos de vulnerabilidad hacemos referencia al carácter de las estructuras e instituciones económicas y sociales y al impacto que provocan éstas en determinados grupos. Por eso expone que la noción de vulnerabilidad social "se refiere a un proceso en el que destacan cuatro dimensiones: trabajo (paro, precariedad, incertidumbre, jubilaciones anticipadas), capital humano (escasa formación, dificultad de acceso a estudios), capital físico (ausencia de recursos productivos) y relaciones sociales (dificultad para formar parte de las redes sociales)" (Pizarro, 2001, pp.14-16).

Lo que nos importa resaltar es cómo, dada su fuerza, el concepto de vulnerabilidad social está siendo muy utilizado como instrumento analítico de situaciones de marginación de todo tipo por su solidez para enfatizar, describir e interpretar esas situaciones. De todas formas, también es verdad que es un término en constante construcción y que se acude a él de manera recurrente para explicar y dar cuenta de situaciones de desventaja socioeconómica y sociocultural de grupos determinados de personas, por ejemplo, inmigrantes o personas con necesidades sociales y educativas concretas.

Así que la exclusión social se entiende como una acumulación de desventajas, de personas y grupos, referidas tanto al trabajo, la vivienda y la educación (las tres clásicas), como a otros factores emergentes (edad, género, salud, cultura, migraciones), por lo que su análisis ha de hacerse desde planteamientos que van mucho más allá que el tradicional de pobreza. Esas desventajas pueden ser debidas al desempleo, pero también a transformaciones estructurales imprevistas. Por eso, Tezanos (2002) hizo un pormenorizado análisis acerca de la inclusión-exclusión. En un esquema que ya es clásico muestra cómo en nuestras sociedades han aparecido, junto a los tradicionales grupos de excluidos, grupos en situación de vulnerabilidad social (cada vez más millones de personas) que forman parte de una 'zona gris' que encontramos entre los círculos de integrados y excluidos. En el círculo central, el de los integrados o incluidos, se encuentran las personas con empleo estable, ingresos suficientes, vivienda propia y que están integrados en redes sociales; el círculo de los excluidos está formado por los desempleados, los que están situados en situación de pobreza, ocupan viviendas muy deterioradas y carecen de apoyos institucionales, incluido en ello la no pertenencia a redes sociales; finalmente, y siguiendo con la imagen de los círculos concéntricos, en medio de los dos anteriores se encuentran las personas vulnerables, con trabajo precario, poco remunerado, vivienda en alquiler, crisis familiares, redes sociales débiles y apoyos institucionales basados en la filosofía compensatoria.

Evidentemente, las situaciones de vulnerabilidad y exclusión repercuten en el bienestar psicológico y, por tanto, en la salud de las personas, sobre todo si atendemos a la declaración constitucional de la Organización Mundial de la Salud de 1948 que, como es sabido, definió la salud como el estado de completo bienestar físico, psicológico y social, definición que ha contribuido a que las ciencias sociales lleven a cabo diferentes estudios del bienestar psicológico como componente esencial y determinante de la calidad de vida. Efectivamente, el concepto de bienestar posee relevancia especial en las áreas de la salud, la situación sociolaboral, la felicidad, las relaciones interpersonales y las relaciones de los seres humanos con el ambiente físico y social, por tanto en el bienestar de las personas. 
Y dentro del estudio del bienestar es particularmente interesante un enfoque cuyo principal marco teórico ha sido construido por Carol Ryff $(1989,1995)$. Esta autora, haciendo una simbiosis entre las teorías del desarrollo humano óptimo, el funcionamiento mental positivo y las teorías del ciclo vital, se refiere al bienestar psicológico o salud mental positiva atendiendo a las siguientes categorías: autonomía, autoaceptación, crecimiento personal, dominio, metas en la vida y relaciones positivas con los otros, buscando una concepción positiva cuyos elementos fundamentales estarían presididos por las metas en la vida y las relaciones interpersonales.

En el campo de la psicología, especialmente en la psicología del trabajo (Duran, 2010), el bienestar psicológico es un constructo que ha sido confundido en muchas ocasiones con otros constructos como felicidad, satisfacción, salud e incluso optimismo, o con expresiones referidas a calidad de vida y la salud mental (Avia y Vázquez, 1998; Blanco y Díaz, 2005; Csikszentmihalyi, 1990; Hills y Argyle, 2002; Seligman, 2002; Seligman y Csikszentmihalyi, 2000; Veenhoven, 1984; 1994; 1995; Warr, 1990); sin embargo, su estudio en las últimas décadas permite una definición que indica que es muy determinante la evaluación que la persona hace de su vida en áreas específicas como la laboral, familiar, de pareja, etc., una evaluación afectiva relacionada con la frecuencia e intensidad de emociones positivas y negativas (Díaz y Sánchez, 2002). De ahí que, según la acepción más comúnmente aceptada, el bienestar (subjetivo o psicológico) sea entendido como un conjunto de juicios valorativos y de reacciones emocionales concernientes al grado en que la propia experiencia es vivida como satisfactoria, agradable y positiva (Andrews y Withey, 1976; Diener, 2000; Diener y Suh, 2001; Diener, Oishi y Lucas, 2003).

En una cultura del bienestar, como en la que estamos inmersos, al menos hasta hace unos años, tienen buena acogida términos relativamente novedosos en psicología, como los de bienestar psicológico subjetivo, satisfacción con la vida o calidad de vida. Por ello, la investigación psicológica de la felicidad o del bienestar subjetivo se ha incrementado notablemente en las últimas décadas, al punto de convertirse en un comportamiento muy estudiado desde la psicología positiva (Alarcón, 2006; Díaz et al., 2006; Diener, 2000; Goñi, Rodríguez y Ruiz de Azúa, 2004; Romero, 2002; SánchezCánovas, 1998; Steel, Schmidt y Shultz, 2008).

Recordaremos que el término bienestar psicológico surgió en Estados Unidos y fue concretándose a medida que la sociedad se fue industrializando y aumentando al tiempo el interés por las condiciones de vida (Garaigordobil, Aliri, y Fontaneda, 2009). Preguntas como ¿por qué algunas personas se sienten más satisfechas con sus vidas que otras? y ¿cuáles serían los factores relacionados con el bienestar psicológico y la satisfacción? dieron origen a muchos de los estudios sobre esta temática. Inicialmente, se asociaba el sentirse bien con la apariencia física, la inteligencia o el dinero, pero actualmente, como resultado de diversas investigaciones en el tema, se sabe que el bienestar psicológico no sólo se relaciona con estas variables sino también con la subjetividad personal (Diener, Suh y Oishi, 1997). Así, Diener (1994) describe tres características del bienestar psicológico: a) su carácter subjetivo, que descansa sobre la propia experiencia de la persona; b) su dimensión global, puesto que incluye la valoración del sujeto en todas las áreas de su vida; y c) la apreciación positiva, ya que su naturaleza va más allá de la mera ausencia de factores negativos. Más tarde, Diener y Diener (1995) propusieron diferentes concepciones del bienestar psicológico: a) el bienestar como satisfacción con la vida, como la valoración del individuo de su propia vida de forma positiva; b) el bienestar como preponderancia de los sentimientos positivos sobre los negativos; y c) la perspectiva filosófico-religiosa que ve la felicidad como una virtud o gracia. Y, como han observado Díaz et al. (2006), durante la última década se han desarrollado numerosas investigaciones (Oramas, Santana y Vergara, 2007; Durán, 2010; Miñano y Martínez, 2011; Palou, 2012) sobre el bienestar que han ampliado considerablemente el mapa conceptual sobre este constructo. De ahí que podamos afirmar que el bienestar psicológico es un elemento que actúa como un indicador positivo de la salud mental.

\section{La inclusión social de los grupos en riesgos de ex- clusión}

Que una sociedad sea justa requiere, como mínimo, la promoción y el desarrollo de las prácticas participativas típicas de una democracia y aprovechar el desarrollo del conocimiento para la mejora económica y cultural de los ciudadanos a través del acceso al trabajo, a la educación, a la información y el respeto activo de la diversidad cultural y lingüística (Frabboni, 2005). Conseguir ese gran objetivo significa que no haya procesos de exclusión social y que apostemos, más que por la integración, por la inclusión porque, normalmente, se confunde la inclusión con la integración y no son lo mismo.

El concepto de integración se utiliza para quienes han sufrido algún proceso de exclusión y se le quiere adaptar al 'ritmo normal' de una sociedad, mientras que la inclusión es un término más global y abarcador que hace referencia a una filosofía de comunidad (Barrio, 2009). La inclusión es un término que implica integración, pero que va más allá pues introduce la necesidad de adaptar las estructuras sociales a la diversidad existente. Se piensa y entiende la diversidad en términos de normalidad, porque lo normal es que seamos diferentes. La inclusión entiende que todos tenemos necesidades particulares, por lo que las intervenciones orientadas a paliar o eliminar la vulnerabilidad y la exclusión social deben dirigirse a todos los ciudadanos y no sólo a sectores considerados como socialmente marginados. Por tanto, la inclusión trasciende la integración, porque ésta se basa en la normalización de las personas con alguna característica que se salga de lo establecido como normal, mientras que la inclusión aparece como un derecho humano indiscutible, un objetivo prioritario para todos los ciudadanos. En definitiva, la inclu- 
sión tiene que ver con todos los ámbitos relacionados con la calidad de vida de las personas (Castell, 2004).

Algunos autores (Echeita, Parrilla y Carbonell i Paris, 2008; Parrilla, 2007; Booth, Ness y Stromstad, 2003) han hecho un esfuerzo de clarificación que nos parece altamente significativo para entender y fomentar la inclusión. De los trabajos de esos autores destacamos las siguientes conclusiones: a) No se debe hablar de inclusión en relación a determinados grupos específicos, sino como un término que engloba a todos, sin distinción. Las políticas 'focalizadas' que se centraban en grupos concretos ya se han demostrado ineficaces. b) La inclusión no debe significar 'admisión' de los nuevos en la comunidad existente, asimilación, sino construcción de una comunidad nueva que aglutine a todos los grupos, los 'antiguos' y los 'nuevos'. c) La participación debe ser el elemento clave en la construcción de comunidades sociales democráticas. d) Fomentar actitudes críticas entre los ciudadanos es elemento importante para la inclusión. e) Se debe ampliar el foco de la inclusión más allá del sistema educativo y trazar vínculos de conexión entre las instituciones educativas y las instancias sociales y políticas.

Por tanto, para que una sociedad llegue a ser realmente inclusiva todos los grupos y personas que la integran deben estar en condiciones de igualdad, sea cual sea su cultura, sus formas de vida, su origen o sus características individuales; esto supone que todos pongamos de nuestra parte y que se combinen muchos esfuerzos educativos, sociales, políticos y económicos.

Para comprobar lo relevante de esta temática, presentamos diferentes investigaciones de profesionales de distintos ámbitos que trabajan por la inclusión social; así, una investigación de la Universidad de Maryland (Hitti, Mulvey y Killen, 2011) revisó la literatura sobre exclusión social con un enfoque basado en la cultura como paradigma de exclusión, este estudio determinó que los factores importantes de exclusión son la etnia, el sexo, las normas y la identidad. En la misma línea de investigación referente a la exclusión social, encontramos un proyecto $\mathrm{I}+\mathrm{D}+\mathrm{I}$ coordinado por las Universidades de Sevilla y Cantabria (Parrilla, Gallego y Moriña, 2009) en el que participaron jóvenes pertenecientes a grupos vulnerables por motivos de cultura, etnia minoritaria, discapacidad y clase socioeconómica. Esta investigación consideró la exclusión como un complejo proceso longitudinal que es construido socialmente, considerando "el transito a la vida activa como uno de los momentos más delicados y vulnerables en los que pueden conculcarse, negarse o limitarse derechos tan básicos como el acceso al empleo, a una vivienda, la interdependencia económica o la posibilidad de entablar relaciones" (p.215).

En esta línea de investigación son interesantes los estudios que abordan la problemática desde la infancia, pues ahí también hemos encontrado factores de exclusión e indicadores referentes a problemas emocionales, como se constata en una investigación realizada en la Universidad de Granada (López, Fernández, Vives y Rodríguez, 2012), que examina los factores de la práctica de crianza y problemas de conduc- ta a 176 niños de educación infantil, entre 4 y 5 años dentro del marco de la interculturalidad, demostrando que hay una mayor presencia de problemas emocionales y de relación con los compañeros en niños inmigrantes. Por ello, trabajar para favorecer el bienestar psicosocial desde edades muy tempranas es aconsejable y no esperar a que los problemas emocionales desemboquen en exclusión social.

Las revisiones de las investigaciones anteriores nos reafirman en la idea de que, si actuamos para acabar con la exclusión sin tener en cuenta los matices de la misma, podemos caer fácilmente en el asistencialismo y el paternalismo, al tratar a todos los excluidos como pobres sin recursos no sólo económicos sino también personales y sociales. Si actuamos así estamos desactivando el potencial que los propios excluidos tienen para salir de esa situación. Debemos enfocar la salida hacia las causas de esa exclusión, en un trabajo que es más psicológico y educativo que de asistencia social, más de educación social y psicología social que de trabajo social, para entendernos desde profesiones distintas aunque no distantes.

Las consideraciones anteriores nos llevaron a realizar el estudio, cuyo objetivo es analizar el bienestar psicológico de los grupos con mayor riesgo de exclusión social, del que damos cuenta en las páginas que siguen.

\section{Método}

En nuestra investigación utilizamos una metodología descriptiva de corte transversal, al objeto de explorar el bienestar psicológico en los grupos con mayor riesgo de exclusión. De acuerdo con Hernández, Pina y Buendía (1997) la metodología por encuesta es la más apropiado para dar respuesta a problemas tanto en términos descriptivos como de relación de variables. Esta metodología es muy apropiada para recoger opiniones de un gran número de personas, en base a los objetivos planteados. A continuación se describen las características de los participantes, el instrumento de recogida de información y el procedimiento llevado a cabo.

\section{Participantes}

El estudio se realizó en la Comunidad Autónoma de Andalucía, concretamente en la provincia de Jaén. Esta provincia se caracteriza por un predominio del sector agrícola seguido de los sectores servicios, industria y construcción. El estudio quedó constituido por 157 personas, de las cuales el $73.3 \%$ son mujeres y el $26.7 \%$ hombres y cuya media de edad fue de $36.81(D T=11.35$; mínima $=17$; máxima $=62)$. En la selección de los participantes se tuvo en cuenta los grupos de exclusión social, empleando un muestreo aleatorio estratificado. Los grupos estudiados quedaron representados de la siguiente forma: jóvenes de 16 a 24 años (18\%), adultos de 25 a 44 años (52.2\%), mayores de 45 años $(23.6 \%)$ y personas de más de 55 años $(6.2 \%)$. 


\section{Instrumento}

El instrumento utilizado en este estudio fue la Escala de Bienestar Psicológico de Sánchez Cánovas (2007), con adecuadas garantías psicométricas de validez y fiabilidad. En este estudio se administró la subescala de bienestar psicólogico subjetivo compuesta por 30 ítems, la cual valora en qué medida los participantes sienten o piensan, desde 1 (Nunca) a 5 (Siempre), en relación a una serie de afirmaciones sobre el bienestar y la felicidad. Por ejemplo, la escala contiene afirmaciones tales como "estoy ilusionado/a", "siento que todo me va bien". El bienestar psicológico subjetivo se refiere a la felicidad, es decir, al afecto positivo, y no a la combinación de afecto negativo y positivo. La escala tiene el sentido de la felicidad o el bienestar, es decir a mayor puntuación mayor percepción subjetiva de bienestar. Con una puntuación máxima de 150 puntos, su interpretación comprende una relación escalonada en la que cuanto mayor es la puntuación obtenida mayor es la percepción subjetiva de bienestar. La consistencia interna de la prueba es muy elevada $(\alpha=.941)$. Este valor no sólo indica una adecuada consistencia interna del instrumento sino que también es similar a la versión original del instrumento que reporta un $\alpha=.935$. Estudios de la validez de criterio han confirmado correlaciones de esta escala con el inventario de Felicidad de Oxford $(r=.89)$.

\section{Procedimiento}

El contacto con los participantes se realizó a través de los servicios de orientación profesional de la comunidad andaluza, donde acudían para demandar asesoramiento socioprofesional y formación, tras solicitar la autorización a los técnicos responsables de las unidades. Se administró a los participantes el instrumento mencionado anteriormente, con la colaboración de investigadores formados para ello, tras solicitar el consentimiento de los sujetos. Los participantes fueron informados que su participación en el estudio era anónima y voluntaria. Los datos obtenidos con la escala de bienestar psicológico subjetivo fueron analizados con el software estadístico SPSS 19.0.

\section{Análisis de datos e interpretación de resultados}

En este apartado se establecen una serie de indicadores acerca del bienestar psicológico subjetivo en los grupos en riesgo de exclusión social, coincidentes con los ítems que componen el cuestionario utilizado. En la Tabla 1 se muestran los resultados de tales indicadores que sugieren que los participantes no tienen buena suerte $(M=2.55 ; d t=1.04)$ y ver cumplidos la mayor parte de sus deseos $(M=2.75$; $d t=1.04)$. Sin embargo si contemplan como importantes su valía como cualquier otra persona $(M=4.34 ; D T=0.96)$ y enfrentarse a su trabajo y sus tareas con buen ánimo $(M=4.24 ; D T=$ 1.01).

Tabla 1. Estadísticos descriptivos.

\begin{tabular}{|c|c|c|c|c|c|c|}
\hline & $\mathrm{N}$ & Mínimo & Máximo & Suma & Media & Desv. típ. \\
\hline Acostumbro a ver el lado favorable de las cosas. & 157 & 1 & 5 & 523 & 3.33 & 1.134 \\
\hline Me siento alegre. & 157 & 1 & 5 & 541 & 3.47 & 1.006 \\
\hline Me siento bien conmigo mismo/a. & 157 & 1 & 5 & 542 & 3.45 & 1.071 \\
\hline Todo me parece interesante. & 157 & 2 & 5 & 536 & 3.46 & 1.083 \\
\hline Me gusta divertirme. & 157 & 1 & 5 & 597 & 3.88 & 1.151 \\
\hline Me siento Jovial. & 157 & 1 & 5 & 569 & 3.67 & 1.157 \\
\hline Busco momentos de distracción y descanso. & 157 & 1 & 5 & 511 & 3.34 & 1.171 \\
\hline He visto cumplidos la mayor parte de mis deseos. & 157 & 1 & 5 & 432 & 2.75 & 1.042 \\
\hline Tengo buena suerte. & 157 & 1 & 5 & 395 & 2.55 & 1.046 \\
\hline Estoy ilusionado/a. & 157 & 1 & 5 & 510 & 3.29 & 1.116 \\
\hline Se me han abierto muchas puertas en mi vida. & 157 & 1 & 5 & 411 & 2.69 & 1.079 \\
\hline Creo que soy una persona con energía. & 157 & 1 & 5 & 594 & 3.81 & 1.048 \\
\hline Duermo bien y de forma tranquila. & 157 & 1 & 5 & 531 & 3.43 & 1.167 \\
\hline Me creo útil y necesario/a para la gente. & 157 & 1 & 5 & 583 & 3.79 & 1.120 \\
\hline Creo que me sucederán cosas agradables. & 157 & 1 & 5 & 504 & 3.29 & 1.123 \\
\hline Creo que como persona (padre, madre, esposo/a, trabajador/a) he logrado lo que quería. & 157 & 1 & 5 & 510 & 3.27 & 1.225 \\
\hline Creo que valgo tanto como cualquier otra persona. & 157 & 1 & 5 & 682 & 4.34 & .966 \\
\hline Creo que puedo superar mis errores y debilidades. & 157 & 1 & 5 & 634 & 4.09 & 1.034 \\
\hline Me enfrento a mi trabajo y mis tareas con buen ánimo. & 157 & 2 & 5 & 662 & 4.24 & 1.012 \\
\hline Me gusta lo que hago. & 157 & 1 & 5 & 582 & 3.78 & 1.133 \\
\hline Disfruto de las comidas. & 157 & 1 & 5 & 627 & 3.99 & 1.059 \\
\hline Me gusta salir y ver a la gente. & 157 & 1 & 5 & 654 & 4.17 & 1.067 \\
\hline Me concentro con facilidad en lo que estoy haciendo. & 157 & 1 & 5 & 607 & 3.87 & 1.020 \\
\hline Creo que, generalmente, tengo buen humor. & 157 & 1 & 5 & 591 & 3.84 & 1.045 \\
\hline Siento que todo me va bien. & 157 & 1 & 5 & 459 & 2.96 & 1.104 \\
\hline Tengo confianza en mí mismo/a. & 157 & 1 & 5 & 578 & 3.75 & 1.110 \\
\hline Me siento contento/a cuando estoy entre la gente. & 157 & 1 & 5 & 625 & 4.03 & .936 \\
\hline Estoy contento/a con mi forma de ser. & 157 & 1 & 5 & 613 & 3.90 & 1.030 \\
\hline Puedo organizar mi tiempo bastante bien. & 157 & 2 & 5 & 587 & 3.84 & .996 \\
\hline Estoy más sano/a que de costumbre. & 157 & 1 & 5 & 564 & 3.59 & 1.176 \\
\hline $\mathrm{N}$ válido (según lista) & 157 & & & & & \\
\hline
\end{tabular}


La media obtenida en bienestar psicológico subjetivo para la muestra total es de $109.74(D T=19.43)$, lo que indica que los sujetos se sitúan en el centil 45 del baremo, caracterizándose por un nivel medio de bienestar psicológico subjetivo. A partir de la media obtenida $(M=109.74)$ se dividió a los participantes en tres grupos y se calcularon los porcentajes; alto (puntuación $>125$ ) con un $23.1 \%$, medio (puntuación entre 125 y 88) con un $60.7 \%$ y bajo (puntuación $<88$ ) con un $16.2 \%$. Los porcentajes indican que la mayor parte de los participantes presentan un nivel 'aceptable' de satisfacción en sus vidas, mientras que el resto de porcentajes muestran muy satisfactorias o poco satisfactorias sus experiencias vitales.

En el Tabla 2 se observa que las mujeres y hombres presentan un nivel medio bienestar psicológico subjetivo. Las mujeres con un $64.7 \%$ de nivel alto, $21.2 \%$ de nivel alto y $14.1 \%$ de nivel bajo. Los hombres con un $50 \%$ de bienestar psicológico subjetivo medio frente al $28.1 \%$ de nivel alto y el $36.8 \%$ de nivel bajo.

Tabla 2. Bienestar psicológico en función del sexo.

\begin{tabular}{llllll}
\hline \multirow{2}{*}{ Sexo } & Hombre & $\begin{array}{c}\text { Bienestar psicológico } \\
\text { subjetivo }\end{array}$ & Total \\
\cline { 3 - 5 } & & alto & medio & bajo \\
\cline { 3 - 5 } & Mujer sexo & $28.1 \%$ & $50.0 \%$ & $21.9 \%$ & $100.0 \%$ \\
& $\begin{array}{l}\text { \% dentro } \\
\text { de sexo }\end{array}$ & $21.2 \%$ & $64.7 \%$ & $14.1 \%$ & $100.0 \%$ \\
\hline Total & Recuento & $23.1 \%$ & $60.7 \%$ & $16.2 \%$ & $100.0 \%$ \\
\hline
\end{tabular}

Con la finalidad de evaluar si existen diferencias significativas en el bienestar psicológico subjetivo en función del sexo, tras comprobar los supuestos básicos, se llevó a cabo la prueba $t$ de student. Los resultados evidenciaron diferencias estadísticamente no significativas en función del sexo ( $F$ [2.454]; Sig.[0.120]; $p<$.05), según se muestra en Tabla 3.

Tabla 3. Prueba $t$.

\begin{tabular}{lcccccc}
\hline & Sexo & $\mathrm{N}$ & Media Desviación & $\begin{array}{c}\text { Error } \\
\text { típica }\end{array}$ & $t$ \\
& & & & $t$ típ. & \\
\hline Bienestar & Hombre & 32 & 108.28 & 22.719 & 4.016 & 0.120 \\
psicológico & Mujer & 85 & 110.29 & 18.165 & 1.970 & \\
\hline
\end{tabular}

En lo que se refiere a los grupos de edad, todos se situaban en el nivel medio de bienestar psicológico subjetivo: el grupo de 16 a 24 años (71.4\%), el grupo de 25-44 (58.3\%), el grupo de $45-54$ años (57.1\%) y el grupo de más de 55 años (62.5\%). Estos porcentajes se muestran en la Tabla 4.

Como se puede observar en la Tabla 5, al comparar los grupos de edad, no existen diferencias significativas en bienestar psicológico general en función del grupo de pertenencia (F [2.339]; Sig.[0.077]; $p<.05)$.
Tabla 4. Bienestar psicológico en función de la edad.

\begin{tabular}{|c|c|c|c|c|c|c|}
\hline & & & \multicolumn{3}{|c|}{$\begin{array}{c}\text { Bienestar psicológico } \\
\text { subjetivo }\end{array}$} & \multirow[b]{2}{*}{ Total } \\
\hline & & & alto & medio & bajo & \\
\hline \multirow[t]{4}{*}{ Grupo } & 16-24 años & $\begin{array}{l}\% \text { dentro } \\
\text { de Grupo }\end{array}$ & $19.0 \%$ & $71.4 \%$ & $9.5 \%$ & $100.0 \%$ \\
\hline & 25-44 años & $\begin{array}{l}\% \text { dentro } \\
\text { de Grupo }\end{array}$ & $25.0 \%$ & $58.3 \%$ & $16.7 \%$ & $100.0 \%$ \\
\hline & 45-54 años & $\begin{array}{l}\% \text { dentro } \\
\text { de Grupo }\end{array}$ & $17.9 \%$ & $57.1 \%$ & $25.0 \%$ & $100.0 \%$ \\
\hline & 55 y más & $\begin{array}{l}\% \text { dentro } \\
\text { de Grupo }\end{array}$ & $37.5 \%$ & $62.5 \%$ & $.0 \%$ & $100.0 \%$ \\
\hline Total & & $\begin{array}{l}\% \text { dentro } \\
\text { de Grupo }\end{array}$ & $23.1 \%$ & $60.7 \%$ & $16.2 \%$ & $100.0 \%$ \\
\hline
\end{tabular}

Tabla 5. ANOVA.

\begin{tabular}{lccccc}
\hline Bienestar psicológico & $\begin{array}{c}\text { Suma de } \\
\text { cuadrados }\end{array}$ & gl & \multicolumn{4}{c}{$\begin{array}{c}\text { Media } \\
\text { cuadrática }\end{array}$} & $F$ & Sig. \\
\hline Inter-grupos & 2561.634 & 3 & 853.878 & 2.339 & .077 \\
Intra-grupos & 41250.674 & 113 & 365.050 & & \\
\hline Total & 43812.308 & 116 & & & \\
\hline
\end{tabular}

\section{Discusión y Conclusiones}

La Psicología Positiva se muestra como un intento por comprender científicamente las fortalezas y potencialidades humanas en contraste con el predominante énfasis que se pone en las alteraciones y trastornos conductuales. Se trata de todo un reto por abordar científicamente el estudio de la felicidad, el optimismo, la esperanza, la sabiduría, la excelencia y la creatividad, lo cual se consigue al investigar determinadas fortalezas y virtudes humanas, así como el efecto de las mismas en las personas y el entorno en el que viven (Carpena y López, 2013).

Cuadra y Florenzano (2003) consideran que esta nueva tendencia psicológica propone potenciar las fortalezas comunes a todas las personas para que funcionen como elementos amortiguadores ante la adversidad y el carácter traumático de aquellas experiencias «penosas» que pueden sobrevenir durante nuestra existencia. El bienestar psicológico no es la vida feliz para siempre sino los procesos de la vida con sus momentos positivos y negativos; una vida que no evita el dolor, sino que se enfrenta a él y le da un significado constructivo para transformar lo negativo en algo digno de vivirse.

Desde esta perspectiva, además de los conceptos de pobreza y vulnerabilidad social podemos adentrarnos en el análisis de la exclusión desde otro concepto: el de riesgo. No de riesgos resultantes de los avatares de la naturaleza o consecuencia de la manipulación de ésta por la especie humana, sino de riesgos sociales, aquellos antes los cuales las poblaciones son socioeconómica y psicológicamente más vulnerables. En este sentido, la vulnerabilidad estaría enmarcada en la probabilidad de ocurrencia de un riesgo, no tanto cuando acaecen pérdidas objetivas en el bienestar social de las personas cuanto que se produzca una percepción subjetiva de 
esa vulnerabilidad, como bien explica Huesca (2003). Ello, evidentemente, tiene repercusión en lo que entendemos por salud mental.

Sin embargo, si tradicionalmente al proceso de la salud mental se accede por el polo negativo, en términos positivos nos remite a lo que se ha nombrado en la literatura específica sobre el tema como psicología positiva o salutogénica. Dentro de esta perspectiva se analizan aspectos como el sentido de coherencia, la fortaleza, la autoeficacia aprendida, el locus de control, la resiliencia, etc. Por eso en nuestro estudio hemos focalizado la atención en el bienestar psicológico, ya que hemos visto que se puede caracterizar la satisfacción y la felicidad como indicadores de buen funcionamiento mental (Oramas, Santana y Vergara, 2007).

Por otra parte si, como se ha demostrado, el bienestar psicológico es un estado integral de salud en el que influyen, además de aspectos psicológicos otros sociales no menos importantes como la situación laboral o las relaciones interpersonales, es evidente que la forma en que se da el proceso depende en gran parte de la persona y de las condiciones y oportunidades concretas que encuentre ésta en el medio sociocultural. El éxito de las personas para alcanzar sus metas depende en gran medida de las estrategias utilizadas y de cómo pueden afrontar los inconvenientes que les plantea al ambiente. El resultado se traduce en bienestar psicológico y calidad de vida o en enfermedades físicas y psíquicas (Castro, 2009).

En relación a esas estrategias, es necesario resaltar que en la última década ha habido muchos programas para la mejora

\section{Referencias}

Alarcón, R. (2006). Desarrollo de una escala factorial para medir la felicidad. Revista Interamericana de Psicología, 40, 99-106.

Barrio, J. L. (2009). Hacia una educación inclusiva para todos. Revista Complutense de Educación, 1 (20), 13-31.

Barton, L. (2008). Superar las barreras de la discapacidad. Madrid: Morata

Booth, T., Ness, K. y Stromstad, M. (2003). (Eds). Developing inclusive teacher education. London: Routledge.

Carpena, A. y López, O. (2013). Emociones positivas, creatividad y problemas de salud en el aula. Navarra: EUNSA

Castel, R. (2004). Encuadre de la exclusión. En S. Karsz (Coord.), La exclusión: bordeando sus fronteras. Definiciones y matices (pp. 55-86). Barcelona: Gedisa.

Castro, A. (2009). El bienestar psicológico: cuatro décadas de progreso. Revista Interuniversitaria de Formación del Profesorado, 66 (23,3), 43-72.

Cuadra, H. y Florenzano, R. (2003) El bienestar subjetivo: Hacia una psicología positiva. Revista de Psicología de la Universidad de Chile, 12 (1), 83-96.

De Haro, R. (2009). Educación intercultural: posibilidades y dificultades para desarrollar una escuela para todos. En A. García (Dir.), El diálogo intercultural (pp. 219-243). Murcia: Editum.

Díaz G. y Sánchez, M. (2002). El bienestar subjetivo. Actualidad y perspectiva. Revista Cubana de medicina General Integral, 17(6), 572-579

Díaz, D., Rodríguez-Carvajal, R., Blanco, A., Moreno-Jiménez, B., Gallardo, I., Valle, C. y van Dierendonck, D. (2006). Adaptación española de las escalas de bienestar psicológico de Ryff. Psicothema, 18, 572-577.

Diener, E. (1994). Assessing subjective well-being: Progress and opportunities. Social indicators research, 31, 103-157.

Diener, E. (1994). El bienestar subjetivo. Intervención Psicosocial, 3, 67-113.

Diener, E. (2000). Subjective well-being: the science of Happiness and proposal for a national index. American Psychologist, 55, 34-43.

Diener, E. y Diener, M. (1995). Cross-cultural correlates of life satisfaction and self-esteem. Journal of Personality and Social Psychology, 68, 653-663. del bienestar psicológico, la mayoría de ellos basados en microteorías o en datos empíricos de líneas de investigación sencillas (Vázquez, Sánchez y Hervás, 2008). Fundamentalmente la estrategia empleada consiste en detectar las variables psicológicas que diferencian a los individuos con alto y bajo bienestar mediante estudios correlacionales y longitudinales, para verificar después igualmente las intervenciones de forma experimental. Por ejemplo, Lyubomirsky (2007) diseñó un programa, con una serie de actividades tendentes a mejorar el bienestar psicológico de las personas que consistía en que éstas contaran cosas buenas que les habían sucedido, escribir cartas de gratitud, etc.

En el caso concreto de nuestro estudio, destacamos que la mayor parte de los sujetos participantes en la investigación presentaron un nivel medio de satisfacción en sus vidas; los resultados de la investigación evidenciaron diferencias estadísticamente no significativas en función del sexo; y al comparar los grupos de edad, resultó que no encontramos diferencias significativas en bienestar psicológico en función del grupo de pertenencia. Por eso, para ahondar en estos estudios, se sugiere continuar las investigaciones sobre el tema, pensando en la integración del modelo sobre bienestar psicológico con algunos otros constructos como la calidad de vida, la salud mental, la autoestima, el proyecto de vida, entre otros, desde el paradigma de la psicología positiva el cual, siguiendo a Lee, Stenn y Seligman (2005), aborda al ser humano desde una perspectiva integradora bajo la cual sea concebido como un agente activo que construye su propia realidad.

Diener, E., Suh, E., Lucas, R. y Smith, H. (1999). Subjective well-being: Three decades of progress. Psychological bulletin, 125 (2), 276-302.

Dolan, S., García, S. \& Díez, M. (2005). Autoestima, estrés y trabajo. Madrid: Mac Graw Hill.

Durán, M.M. (2010). Bienestar Psicológico: el estrés y la calidad de vida en el context laboral. Revista Nacional de Administración, 1(1), 71-84

Echeita, G., Parrilla, A. y Carbonell i París, F. (2008). Hacia un marco de referencia común para la educación del alumno en desventaja. La educación especial a debate. Ponencia-Marco en XXV Jornadas de Universidades y Educación Especial. Universidad de Vic. 12 y 13 de marzo de 2008.

Frabboni, F. (2005). Societá della conoscenza e scuola. Trento: Erikson.

Garaigordobil, M., Aliri, J. y Fontaneda, I. (2009). Bienestar psicológico subjetivo: diferencias de sexo, relaciones con dimensiones de personalidad y variables predictoras. Behavioral Psychology, 17(3), 543-559

Goñi, A., Rodríguez, A. y Ruiz de Azúa. S (2004). Bienestar psicológico y autoconcepto físico en la adolescencia y juventud. Psiquis 25, 141-151.

Herrera, J. (2005). Los derechos bumanos como productos culturales. Crítica del bumanismo abstracto. Madrid: Los libros de la Catarata.

Hessel, S. (2011). Indignaos. Barcelona: Destino.

Hervás, G. (2009). Psicología positiva: una introducción. Revista Interuniversitaria de Formación del Profesorado, 66, 23-41.

Hitti, A., Mulvey, K. L. and Killen, M. (2011). Social exclusion and culture: the role of group norm, group identity and fairness. Anales de Psicologias, 27 (3), 587-599.

Huesca, A. (2003). Sociedad del riesgo y desigualdad social. Jornadas sobre prevención de catástrofes. Foro Euromediterráneo. Madrid, 6-8 octubre.

Kennedy, D. E. \& Kramer, L. (2008). Improving emotion regulation and sibling relationship Quality. The more fun with sisters and brothers program. Family Relations, 57 (5), 567-578. 
Kevin, M. D. (2009). Meternal repors of marital quality and preschoolers' positive per relations: the moderating roles of effortful control, positive emotionality, and gender. The Journal of positive Psychology, 4 (1), 168-181.

Keyes, C. L. M. y López, S. J. (2002). Toward a science of mental health: positive directions in diagnosis and interventions. En C. R. Snyder y S. J. López (Dirs.), Hadbook of positive psychology (pp. 45-59). Londres: Oxford University Press.

Lee, A., Stenn, T., y Seligman, M. (2005). Positive psychology in clinical practice. Annual Review of Clinical Psychology, 1, 629-651.

López, S., Fernández, A., Vives, M ${ }^{\text {a }}$ C. y Rodríguez, O. (2012). Prácticas de crianza y problemas de conducta en niños de educación infantil dentro de un marco intercultural. Anales de Psicología, 28 (1), 55-65.

Lyubomirsky, S. (2007). The how of happiness. New Cork. The Penguin Press.

Miñano, L. y Martínez, S. (2011). El asociacionismo y las necesidades socioeducativas en los centros de mayores del municipio de Murcia. Revista de Investigación Educativa, 29 (2), 325-340.

Oramas, A., Santana, S. y Vergara, A. (2007). El bienestar psicológico, un indicador positivo de la salud mental. Revista Cubana de Salud y Trabajo, 7 (1-2), 34-39.

Palou, B. (2012). Análisis de los elementos configurativos de la ciudadanía como condición para la integración de la juventud de origen magrebí en Cataluña. Revista de Investigación Educativa, 30 (1), 181-195.

Parrilla (2007). Inclusive Education in Spain: a view from inside. En L. Barton y F. Armstrongs (Eds). Policy, Experience and Change: Cross Cultural Refletions on Inclusive Education (pp. 19-36). Dordrecht: Springer Ed.
Parrilla, A., Gallego, y Moriña, A. (2009). El complicado tránsito a la vida activa de jóvenes en riesgo de exclusión: una perspectiva biográfica. Revista de Educación, 351, 211-233.

Pizarro, R. (2001). La vulnerabilidad social y sus desafios: una mirada desde América La tina. Santiago de Chile: CEPAL, Publicaciones de Naciones Unidas.

Peterson, C. (2006). A primer impositive psychology. New York: Oxford University Press.

Portera, A. (2006). Globalizzarione e Pedagogia interculturale. Trento: Erikson.

Romero, E. (2002). Investigación en Psicología de la personalidad. Líneas de evolución y situación actual. Boletín de Psicología, 74, 39-77.

Ryff, C. (1989). Happiness is everything, ori s it? Exploration on the meaning of psychological web-begin. Journal of Personality and Social Psycology, 57, 1069 1081

Ryff, C. y Keyes, C. (1995). Th estructure of psychological well-being revised. Journal of Personality and Social Psycology, 69, 719-727.

Sánchez-Cánovas, J. (2007). EBP. Escala de Bienestar Psicológico. Madrid. TEA.

Seligman, M. E. P. \& Csikszentmihalyi, M. (2000). Positive Psychology: an introduction. American Pychologist, 55 (1), 5-14.

Steel, P., Schmidt, J. y Shultz, J. (2008). Refining the relationship between personality and subjective well-being. Psychological Bulletin, 134, 138-161.

Tezanos, J. F. (2002). Desigualdad y exclusión social en las sociedades tecnológicas. Revista del Ministerio de Trabajo y Asuntos Sociales, 35, 35-54.

Vázquez, C., Sánchez, A. y Hervas, G. (2008). Intervenciones psicológicas para la promoción del bienestar. En C. Vázquez y G. Hervas (Eds.), Psicología Positiva Aplicada (pp. 41-71). Bilbao: Desclee de Brower.

(Articulo recibido: 24-7-2012; revisado: 11-2-2013; aceptado: 27-2-2013) 